\title{
CALLING CELLS TO ARMS
}

Increased understanding of immune-and tumour-cell biology has led to an explosion of research into potential ways to harness the immune system to kill cancer. By Emily Elert.

\section{NATURAL IMMUNE RESPONSE}

The body's specific immune response begins when specialized immune cells present pieces of foreign matter, called antigens, to immature immune cells in the lymph nodes.

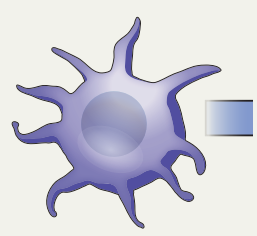

1 Roaming dendritic cell (DC) captures and processes foreign material.

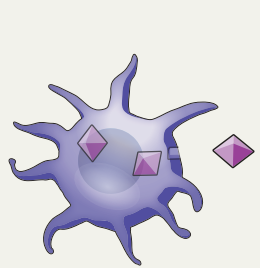

2. DC presents foreign antigen to immature T cell.

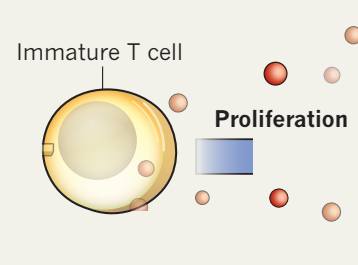

(3) Activated $T$ cell releases cytokines to stimulate immune response, including $\mathrm{T}$ cell proliferation and maturation.

\section{Effector T cell}

Stimulates proliferation of B cells, which produce antibodies.

\section{THERAPEUTIC APPROACHES}

Current cancer immunotherapies can be broken down into three major types: non-specific therapies, monoclonal antibodies and vaccines.

\section{NON-SPECIFIC IMMUNOTHERAPIES}

These drugs include cytokines and other chemicals that stimulate a general immune response. Most likely to be used as adjuvants to other therapies, such as vaccines.

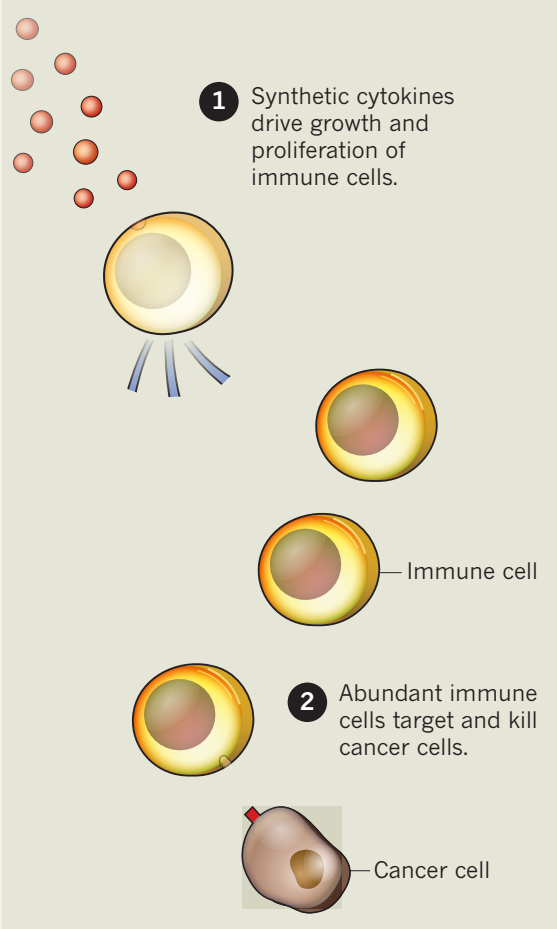

\section{MONOCLONAL ANTIBODIES}

These proteins stick to specific antigens, either directly affecting cells or tagging them for destruction. Uses include:

Carrying drugs or toxins to target cells.
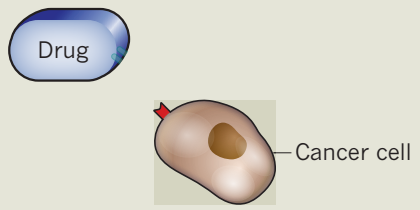

$\mathrm{OR}$

Tagging cell for destruction by immune cells.

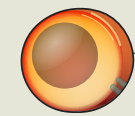

Killer immune cell

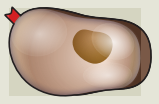

OR

Blocking signalling pathway to halt growth or proliferation.

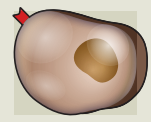

\section{VACCINES}

Vaccines are made from cancer cells, parts of cells or antigens designed to stimulate the immune system to attack a tumour. Multiple approaches are being tested, including DC vaccines.

(1)

A patient's DCs are removed, $\quad-$-Antigen stimulated and re-infused with a cancer-specific antigen.
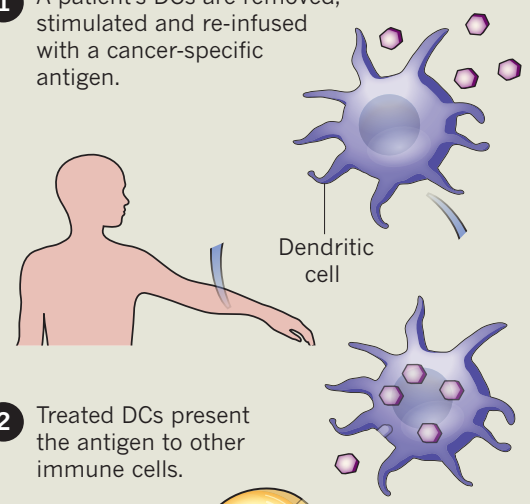
immune cells.
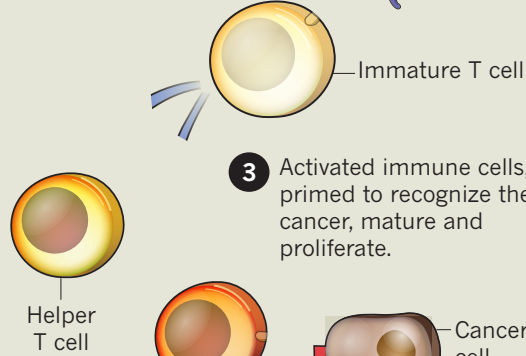

3 Activated immune cells primed to recognize the cancer, mature and proliferate.

T cel
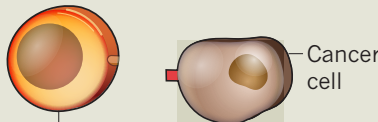


\section{SEARCHING FOR SYNERGY}

Single immunotherapies have been only modestly effective, so researchers are searching for synergistic combinations of drugs. The ideal attack below includes existing therapies, drugs in clinical trials and theoretical compounds.

(1) Tumour microenvironment

Initial therapy increases vulnerability of cancer cells to immune attack.

Drugs block

anti-apoptosis pathways in tumour cells and inhibit regulatory $\mathrm{T}$ cells.

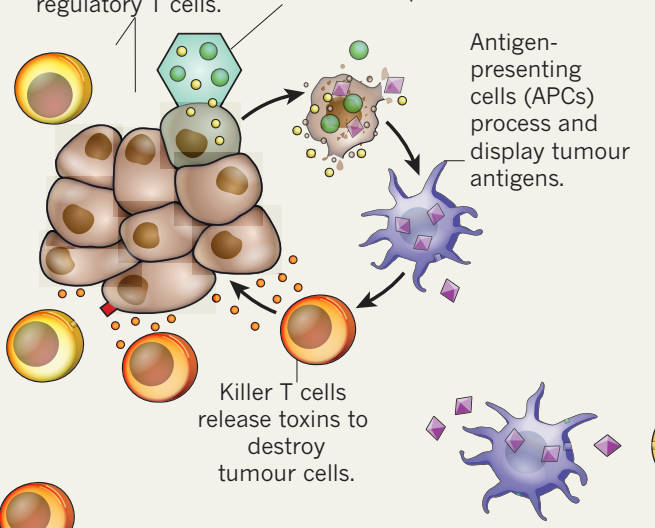

Modified virus invades cancer cells and causes cell lysis, eliciting innate mmune response.

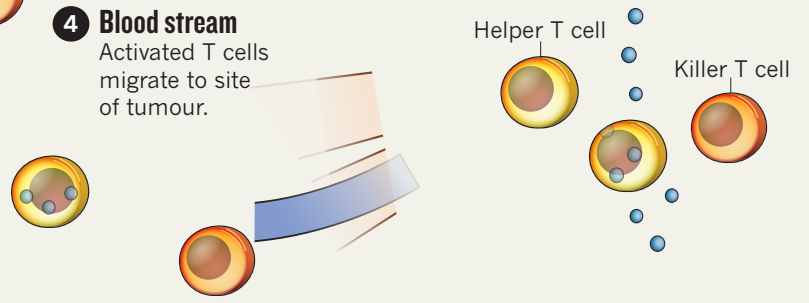

Vaccine

Cancer-specific antigens, non-specific adjuvants, and molecules to increase immune activity.
Cytokines activate T cells

$$
000
$$$$
\text { - } 00
$$

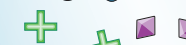

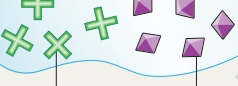
APC mobilizers activate APCs antigen

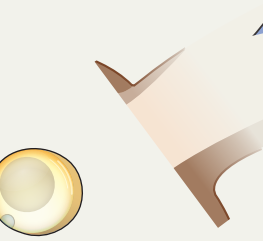

\section{IMMUNOTHERAPIES ON TRIAL}

The number of cancer immunotherapies in phase III clinical trials has risen sharply since the early 1990s, reflecting renewed interest in immune-based cancer treatments among researchers and drug-makers.

Monoclonal antibodies (157 trials)

Non-specific immunotherapies (269 trials)

Vaccines (113 trials)

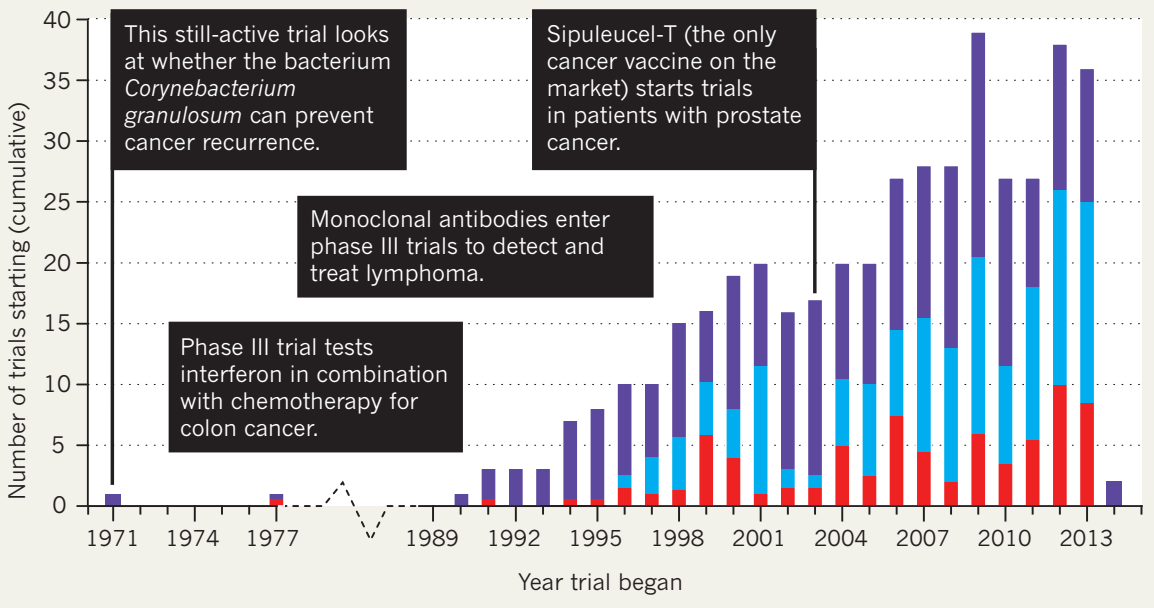

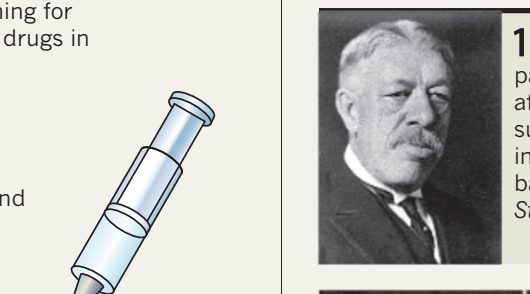

\section{Lymph nodes}

APCs present vaccine antigens to immature T cells, which mature and proliferate.

\section{A DURABLE CONCEPT}

1891 After reading that a patient's tumour disappeared after a bacterial infection, surgeon William Coley begins jecting cancer patients with bacteria (now known to be Streptococcus pyogenes).
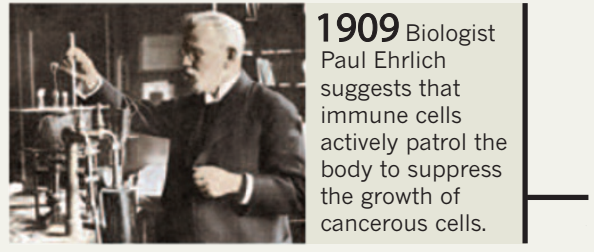

1953 Mice show immunity to tumour cells after cancer is allowed to grow and then surgically removed, suggesting the existence of tumour-specific antigens.

1957 Discovery of interferon, an immunestimulating cytokine that will eventually be used as a non-specific cancer immunotherapy.

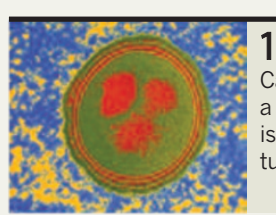

1959 Bacillus Calmette-Guérin (BCG), a tuberculosis vaccine, is shown to inhibit tumour growth in mice.

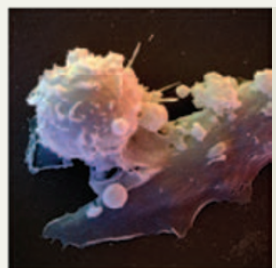

1973 Dendritic cells described by Ralph Steinman and Zanvil A. Cohn Steinman won the Nobel prize for this discovery.

1983 Discovery of T-cell antigen receptors, which detect antigens presented by other immune cells and ramp up the immune response.
1986 First humanized antibodies approved by the US Food and Drug Administration (FDA).

Combination treatment with ipilimumab and nivolumab, a PD-1 blocker, reduces tumour size in melanoma.

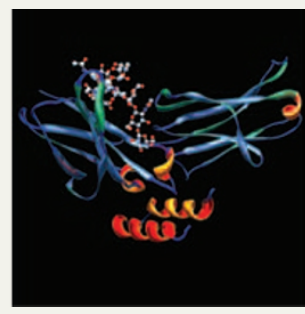

1997 The first monoclonal antibody for cancer, Rituximab, approved by the FDA for treating non-Hodgkin's lymphoma.
2008 First therapeutic cancer vaccine Oncophage, wins approval in Russia for treating kidney cancer.

2010 FDA approves Dendreon's cancer vaccine, Provenge (sipuleucil-T), for the treatment of prostate cancer. 